\title{
Variables predictoras de riesgo frente a los derechos del infante en la era digital. Un estudio de México y España.
}

\section{Risk predictive variables against the rights of the infant in the digital age. A study made in Mexico and Spain}

\author{
iD Carlos René Contreras Cázarez ; carlos.contreras@unison.mx \\ iD Gustavo Adolfo León Duarte ${ }^{1}$ gustavo.leon@unison.mx \\ D Luisa Dolores Zozaya Durazo²; Izozaya@alumni.unav.es
}

\begin{abstract}
Resumen
Los dispositivos móviles inteligentes son herramientas útiles para comunicarse y entretenerse. Sin embargo, como cualquier instrumento o actividad, tienen sus peligros, amenazas y debilidades, especialmente para los más indefensos: los niños. Este artículo discute la socialización, la exposición al riesgo y la dependencia de Internet frente a los derechos fundamentales de la infancia intermedia en el uso de dispositivos móviles. Implementa una metodología descriptivo-correlacional para exponer la prueba de validez de constructos teóricos disciplinares y su relación empírica explicativa mediante un análisis de regresiones. Se aplicó un cuestionario a 4 escalas a 275 estudiantes de educación primaria en España y México. Se utilizó el programa estadístico SPSS, versión 23, y el programa EQS versión 6.1, obteniendo datos descriptivos, pruebas de fiabilidad, correlaciones y un modelo estructural. Los hallazgos confirman que la dependencia de Internet propicia, entre otros factores de riesgo, la exposición al contenido sexual de los menores y vulnera sus derechos constitucionales y universales del infante.
\end{abstract}

Palabras clave: Educación, riesgos, derechos del niño, Internet, dependencia.

\begin{abstract}
Smart mobile devices are useful tools for communicating and entertaining. However, like any instrument or activity, it has dangers, threats and weaknesses, especially for the most defenseless: The children's. This article discusses socialization, risk exposure and Internet dependence on the fundamental rights of intermediate children in the use of mobile devices. It implements a descriptive-correlational methodology to expose the validity test of disciplinary theoretical constructs and their explanatory empirical relationship through a regression analysis. A 4-scale questionnaire was applied to 275 primary school students in Spain and Mexico. The statistical program SPSS, version 23, and the program EQS version 6.1 were used, obtaining descriptive data, reliability tests, correlations and a structural model. The findings confirm that Internet dependence promotes, among other risk factors, exposure to sexual content of minors and violates their constitutional and universal rights of the infant.
\end{abstract}

Keywords: Education, risks, children rights, Internet, dependence.

\footnotetext{
${ }^{1}$ Universidad de Sonora

2 Universidad de Navarra España
} 


\section{INTRODUCCIÓN}

Las relaciones entre la exposición al riesgo, la socialización y la dependencia de Internet en jóvenes han sido ampliamente abordadas por separado y desde diferentes perspectivas de las ciencias sociales, suscitando sugestivos debates éticos y epistemológicos. La presente investigación remite, fundamentalmente, al impacto que tienen determinadas variables latentes y manifiestas, así como factores de riesgo, de socialización y de dependencia de Internet frente a los derechos fundamentales de la infancia intermedia en el uso de dispositivos móviles.

Según el Fondo de las Naciones Unidas para la Infancia (UNICEF) (2017) los menores que han crecido inmersos en la denominada era digital, por una parte, fortalecen lazos de amistad y desarrollan experiencias de aprendizaje, incrementando sus ventajas en una sociedad basada en el conocimiento; pero por otra parte, se vincula a peligros en línea, siendo el núcleo central de las preocupaciones generalizadas sobre las amenazas que enfrentan los niños y niñas en internet; exponiéndolos a un contenido inaceptable, a un comportamiento inadecuado y a contactos potencialmente peligrosos con el mundo exterior.

Algunos estudios refieren a conductas y acciones encaminadas a potencializar el acoso, el hostigamiento y la exposición a la pornografía (UNICEF, 2014; 2017, 2018). Otras investigaciones en México y España muestran que durante los periodos 2013-2014 y 2014-2015 los jóvenes entre 12 y 15 años de edad accedían de $83 \%$ a 84.5\%, incrementándose poco menos de $1.5 \%$ entre cada diagnóstico, según el estudio presentado por León, Contreras y Moreno (2016); León, Caudillo, Contreras y Moreno, (2014a, 2015b). Por otra parte, y en este mismo contexto, el estudio presentado por Contreras (2019) evidenció a través de un modelo estructural una tendencia de acceso y consumo de internet en menores con fines de socialización. Los datos descriptivos del estudio revelaron que más del $90 \%$ de los menores encuestados utiliza la telefonía celular inteligente para sus procesos de socialización e interacción con sus iguales.

\subsection{Prevalencias de la población y sujetos de estudio con conexión a Internet (España y México).}

En España, para el cierre de 2017, el 85\% de los españoles contaban con acceso a Internet, mientras que en 2006 solo accedían el 38\%. Los datos se duplicaron en una década. En el caso de los datos de menores de edad, estos van referidos a partir del año 2007 y directamente dirigido al colectivo de 10 a 15 años.

En México, de acuerdo con el Instituto Nacional de Estadística y Geografía, INEGI (2018), existían 74.3 millones de usuarios de Internet de seis años o más, lo que representa el $65.8 \%$ de la población en ese rango de edad. Para este año, el 73.5\% de las y los mexicanos de seis años o más utilizó el teléfono celular (Smartphone). En este país, los estados con una mayor proporción de usuarios en áreas urbanas fueron Sonora, Baja California, Quintana Roo y Nuevo 
León, con $83.3 \%$, 81.7\%, 80.3\% y 79.8\% respectivamente. La ciudad mexicana con mayor índice de conexión y mayor proporción de usuarios de Internet es Hermosillo, Sonora.

\subsection{Meta científica.}

Como bien se sabe, una teoría científica puede definirse como un conjunto lógicamente conectado de afirmaciones abstractas a partir de las cuales se puede derivar algún tipo de hipótesis y explicaciones empíricamente comprobables (Craig, 2013). En esta investigación se parte de una hipótesis predictiva general: el uso excesivo de Internet puede agravar los riesgos del menor de edad al estar en línea y desarrollar, adicionalmente, experiencias negativas en la infancia intermedia pese a los derechos universales que el menor de edad tiene.

Específicamente, el presente artículo tiene como meta científica analizar y explicar empíricamente la exposición al riesgo en la infancia intermedia al hacer un uso excesivo de Internet mediante la utilización de dispositivos móviles. El reto adicional que exponemos es relacionar, comprender y predecir estadísticamente la ocurrencia empírica en dichas variables (socialización, dependencia de Internet y exposición al riesgo) frente a los derechos fundamentales del infante en la era digital, particularmente en el uso de dispositivos móviles.

\subsection{Una cuestión ontológica. Sociabilidad virtual}

El proceso de socialización ha constituido uno de los principales objetos de estudio de las ciencias sociales. Sus aportaciones devienen desde diferentes enfoques conceptuales y metodológicos (Simkin y Becerra, 2013). Desde la perspectiva académica, la sociabilidad es un concepto que tiene sus orígenes en los estudios sociológicos, entre los que se destacan los realizados por autores como Georg Simmel, Max Weber y Georges Gurvitch, citados en Chapman (2015). Para Simkin y Becerra (2013), la socialización puede ser entendida como el proceso mediante el cual los individuos incorporan normas, roles, valores, actitudes y creencias a partir del contexto sociohistórico en el que se encuentran insertos. Cuando se habla de socialización virtual o en línea, se hace referencia a los procesos de interacción por medio de procesos y actividades de comunicación e interacción digital. En este sentido, los conceptos de comunicación, socialización e interacción están estrechamente vinculados. A menudo, de manera práctica dicha relación de integración viene dada por las tecnologías de la información y la comunicación, en especial con Internet, redes sociales y dis positivos tecnológicos.

Desarrollando más esta idea Contreras y Campa (2017), expresa que las redes sociales tienen la capacidad de crear comunidades virtuales, propiciando no solo el espacio idóneo para la interacción y los procesos de sociabilidad entre estudiantes y sus pares, sino también para la interacción con sus padres, profesores, tutores, personas conocidas y desconocida (Contreras, 2019). 
Por su parte otros autores, como Gómez y Redondo (2011) en Ibáñez y Sánchez (2019) relacionan la importancia de iniciar la aproximación a las redes sociales en edades tan tempranas, con "la necesidad de enseñar al alumnado las consecuencias que pueden tener sus actuaciones con las redes sociales, por lo que se les debe de ofrecer unas pautas de comportamiento" (p.10). Según los especialistas, la finalidad de acercar a los menores en etapa de infancia intermedia al uso de estas herramientas es con la finalidad de que se haga un buen uso de ellas, ya que no todo son ventajas al poder presentarse determinados riesgos asociados a su utilización, debido a un uso descontrolado e inadecuado por los más jóvenes (Del Moral y Villalustre, 2012, p.42).

En consecuencia, la evidencia científica ha propuesto y probado algunos modelos teóricos específicos sobre socialización a partir de estudios de correlación, donde se describen, analizan y se correlacionan variables que explican y afectan a otras en un modelo inclusivo interdisciplinario sobre el uso, socialización (Contreras y Campa, 2017) y la dependencia de Internet en jóvenes (León, et al. 2016). En esta misma línea, existen otros modelos teóricos que explican una tendencia de acceso y uso de Internet para fines de interacción y socialización por parte de los menores (Contreras, 2019).

\subsection{Derechos fundamentales de la infancia en la era digital}

Los derechos del niño(a) son concebidos como instrumentos eficaces para liberar al niño de coacciones ancladas en las costumbres o las leyes y proponer el reconocimiento de su pleno status como ciudadano y, por tanto, el disfrute, entre otros, de derechos políticos y sociales (Lozano, 2016). Con el uso de Internet y con el apoyo de las tecnologías se ha contribuido significativamente al desarrollo de los derechos humanos y al mismo tiempo, se ha difundido el conocimiento propiciando el acceso al mismo en sectores de la población mundial al que no se había tenido acceso. Sin embargo, el acceso a las tecnologías y el Internet, particularmente, no solo han originado una serie de situaciones y circunstancias que benefician los derechos fundamentales de los menores de edad, sino que han propiciado una serie de factores e indicadores específicos que definen las conductas y experiencias que presenta en línea (UNICEF, 2005; 2017). Algunos académicos argumentan de igual manera que, Internet, como tal, puede ser una herramienta de ayuda en la protección y el acceso a otros derechos humanos, así como contribuir al crecimiento económico, social y político, beneficiando a la humanidad en su conjunto (Byrne, Kardefelt-Winther, Livingstone, Stoilova, 2016; Fernández, 2016). También se advierte sobre los efectos negativos con relación a los derechos fundamentales del menor de edad. Por ejemplo, para Fernández (2016), el acceso a Internet si bien es un derecho universal, puede afectar a los menores en relación con los contenidos que pueden circular por esta herramienta mientras navegan en solitario. 


\subsection{Dependencia de Internet y exposición al riesgo.}

La literatura científica toma como punto de partida la definición propuesta de adicción establecida por factores e indicadores del Manual of Mental Disorders (DSM) de Bergmark, Bergmark y Findahl (2011). Existe, por otra parte, una falta de consenso por los especialistas en cuanto a la definición de Dependencia de Internet (DI) al día de hoy. En la actualidad, sólo por citar algunos estudios en las últimas dos década, diversas investigaciones refieren a la DI, bajo diferentes nombres: Internet Addiction Disorder (IAD), el uso compulsivo de Internet Compulsive Internet Use (CIU)-o uso patológico de Internet -Pathological Internet Use (PIU), (Davis, 2001; Fortson, Scotti, Chen, Malone y Del Ben, 2017; Kuss y Lopez, 2016; Lin y Tsai, 2002; Montag y Reuter, 2017; Young, 1998, citado en Contreras y León, 2019). De la misma manera, las investigaciones presentadas dan cuenta, no solo de un perfil característico en el uso excesivo de Internet y la relación con las competencias digitales de los adolescentes (Contreras, 2019), sino también de diversos modelos teóricos propuestos para el estudio a partir de variables que influyen y predicen a otros factores de riesgo en el individuo en la DI.

Por otro lado, algunos estudios recientes revelan que la DI puede analizarse, entre otros aspectos, por el uso excesivo de esta herramienta en menores de edad (Rojas, Ramos, Pardo y Hernández, 2018). Igualmente se afirma que ello podría ocasionar trastornos o deterioro en el individuo tanto emocional como psicológico (Vondrácková y Gabrhelík, 2016). En tanto, Echeburúa y Corral $(2009 ; 2010)$ señalan que cualquier conducta rutinaria que parezca placentera es idónea de convertirse en un comportamiento adictivo. En este sentido, tratando de elaborar una salida más holística e integrativa, estos últimos estudiosos precisan que una conducta es adictiva no por la frecuencia con que se realiza sino, más bien, en relación a la dependencia que se crea: a la pérdida de control por parte del sujeto, y la grave interferencia que genera en las relaciones familiares, sociales y laborales. Adicionalmente se incorporan para la integración de la escala las aportaciones europeas recientes, aquellos estudios que han evidenciado una tendencia centrada en los niveles y grados de consumo de contenidos pornográficos, así como contenidos violentos y racistas como parte del acceso en los menores (Livingstone, Haddon, Görzig y Ólafsson, 2017; Livingstone, Mascheroni y Staksrud, 2018).

\section{Método}

\subsection{Enfoque y diseño de la investigación}

El presente informe se abordó desde el enfoque hipotético-deductivo con fundamento en el paradigma positivismo. El estudio es de tipo descriptivo-correlacional; así, además de especificar propiedades y características de las variables analizadas, es posible establecer correlaciones entre las mismas. Se utilizó un diseño no experimental para la investigación de tipo transversal, por lo que las variables del presente estudio se analizaron en su contexto real (Hernández, Fernández y Baptista, 2010), sin la manipulación deliberada, y en un momento único de recolección de datos (2017-2018). 


\subsection{Participantes del estudio.}

Debido a las prevalencias de la población objetivo mencionadas con anterioridad, el cuestionario se aplicó a estudiantes de 5 to y 6 to año de educación primaria ubicados en las ciudades de Madrid, España y Hermosillo, México. La muestra está conformada por

275 menores. En el contexto europeo, el colegio seleccionado fue la escuela primaria IdeoMadrid, ubicada en la carretera de Colmenar a Alcobendas, Km. 0,500, en la ciudad de Madrid, España. En el contexto mexicano las instituciones educativas en las que se aplicó el cuestionario fue la escuela primaria Enrique Rebsamen y la primaria Ignacia Fimbres. Ambas escuelas ubicadas en la ciudad de Hermosillo, Sonora, México. La selección de las instituciones educativas se realizó con base a los altos niveles de conectividad de Internet y el número de matrícula de estudiantes en cada uno de los centros.

\subsection{Instrumento}

Se elaboró un instrumento cuantitativo conformado a 4 escalas: exposición al riesgo, dependencia de internet, sociabilidad virtual y derechos fundamentales. La escala de exposición al riesgo se diseñó para el presente estudio. Está conformada por 16 reactivos que analiza el acceso involuntario a páginas con contenido potencialmente nocivos -no apropiados para menores de edad como: pornografía, violencia y situaciones de humillación hacia otras personas o hacia uno mismo. La escala dependencia de Internet se retomó del modelo inclusivo interdisciplinario de dependencia de Internet por León, et al. (2016), citado en Contreras (2019) conformado por 16 ítems, el cual mide el apego a los dispositivos tecnológicos móviles. En tanto, la escala de sociabilidad virtual está conformada por 25 ítems (Contreras, 2019; 2020) los participantes debían responder acerca de sus hábitos de consumo en redes sociales y espacios que emplean para la interacción virtual con sus pares, padres, personas conocidas y desconocidas. Por último, la escala de los derechos fundamentales de los menores agrupada con 23 reactivos; analiza los derechos fundamentales de estudiantes de primaria y secundaria en el uso y dependencia de Internet en la sociedad de la información; tales como el acceso a la información, libre expresión y asociación, protección a la intimidad (Contreras, 2020).

Para cada uno de estos reactivos, el participante debía responder al instrumento con base a una escala Likert con 5 alternativas de respuesta, donde $1=$ nunca, 2 = rara vez, $3=$ algunas veces, 4 = casi siempre y 5 = siempre.

\subsection{Procesamiento, análisis y validación del instrumento.}

Para el procesamiento de los datos, la información se sometió al programa estadístico SPSS, versión 23. Se realizó un análisis estadístico descriptivo y para estimar la confiabilidad del instrumento, se calcularon las alfas de Cronbach eliminando el elemento más bajo (Cronbach, 1984) para medir la consistencia interna de cada una de las escalas que componen el cuestionario, obteniendo alfas considerables con coeficientes aceptables. El análisis permitió igualmente, obtener las estadísticas univariadas de tendencia central: media, mediana, moda 
y desviación estándar. Seguidamente, se elaboraron las parcelas correspondientes con los ítems de las escalas. Por medio de un análisis de factores principales con el método de rotación varimax propuesta por Kaiser (1958, en Contreras y León, 2019) fue posible establecer las parcelas de todos los factores que componen el modelo estructural propuesto para esta investigación.

El análisis factorial confirmatorio (AFC) que se realizó por medio del programa EQS, versión 6.1, permitió establecer las correlaciones del modelo estructural propuesto. Mediante el análisis estadístico multivariado, se probó un modelo estructural por medio de factores que se relacionan y componen al modelo.

\section{RESULTADOS}

\subsection{Estadísticos descriptivos}

la tabla 1 muestra las frecuencias de las variables demográficas. La muestra total fue de 275 estudiantes de educación primaria, de los cuales el $42 \%$ representa al sexo masculino y un $58 \%$ al sexo femenino. El rango de edad de los menores participantes fue de 10 a 13 años, con una media de 10.85 y una desviación estándar de .086.

Tabla 1. Frecuencias de las variables demográficas de los estudiantes de educación primaria y secundaria.

\begin{tabular}{cccc}
\hline Variable & & Frecuencia & Total \\
\hline Sexo & Mujer & 148 & \\
& Hombre & 127 & 275 \\
Edad & 10 & 66 & \\
& 11 & 77 & \\
& 12 & 80 & \\
Grado escolar & 13 & 52 & 275 \\
& 5 to & 143 & \\
& 6to & 132 & 275 \\
\hline
\end{tabular}

La Tabla 2 muestra el análisis de las escalas que conforman el instrumento, donde es posible apreciar que cada una de ellas resultó con un alfa de Cronbach superior a .60, por lo que se asume que las mismas presentan un considerable grado de confiabilidad. 
Tabla 2. Alfas de las escalas e ítems que conforman el instrumento. Fuente: Contreras (2020).

\section{Escala/ítem}

Exposición al riesgo

Cuando uso Internet me aparecen videos con contenido para adultos.

Me molesta ver páginas con contenido para adultos.

Me divierte ver videos de personas haciendo bromas a otras personas.

Me gusta ver videos con contenido para adultos cuando nadie me ve.

Me incomoda ver imágenes o videos de personas con contenido para adultos

Me gusta ver videos de peleas entre estudiantes cuando estoy en Internet.

Cuando estoy solo en casa, busco videos o imágenes con contenido para adultos

Me divierte ver videos de personas hacienda bromas pesadas a otras.

Cuando uso Internet busco información para mis tareas escolares.

Cuando uso Internet es solo para redes sociales.

Cuando uso Internet es solo para correo electrónico (e-mail).

Cuando uso Internet se abren páginas para adultos accidentalmente.

Es divertido ver páginas con contenido para adultos cuando nadie me ve.

Me divierte ver videos de personas discutiendo y peleando.

Comparto fotos o videos de bromas pesadas por redes sociales.

Envío memes por redes sociales a mis amigos

\section{$N$ Min. Max. Media D.E. Alfa}

Sociabilidad virtual aplicaciones

Hablo con mis compañeros de clase por redes sociales sobre tareas que dejan en la escuela.

Me pongo de acuerdo por redes sociales con mis amigos para salir de paseo

Me comunico con familiares por redes sociales

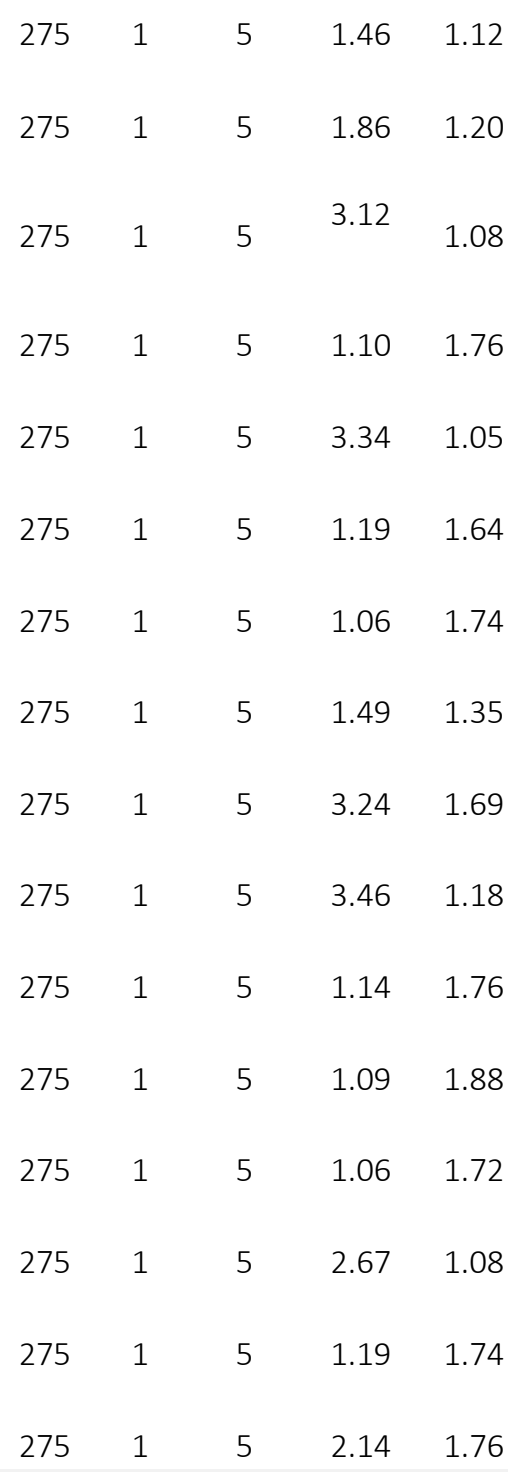

$\begin{array}{lllll}275 & 1 & 5 & 3.37 & 1.18\end{array}$

\begin{tabular}{lllll}
275 & 1 & 5 & 2.24 & 1.08 \\
275 & 1 & 5 & 1.21 & 1.03 \\
275 & 1 & 5 & 1.34 & 1.12 \\
\hline
\end{tabular}

\section{9}

Página $129 / 139$

DOI: https://doi.org/10.21556/edutec.2020.73.1549 


\begin{tabular}{|c|c|c|c|c|c|c|}
\hline Escala/Ítem & $N$ & Min. & Max. & Media & D.E. & Alfa \\
\hline Uso redes sociales para enterarme de lo que pasa en la escuela. & 275 & 1 & 5 & 1.48 & 1.16 & \\
\hline Uso redes sociales para conocer nuevos amigos. & 275 & 1 & 5 & 2.18 & 1.09 & \\
\hline Me gusta conocer a nuevas personas por redes sociales. & 275 & 1 & 5 & 1.33 & 1.02 & \\
\hline $\begin{array}{l}\text { Uso redes sociales y aplicaciones para hablar con personas que } \\
\text { no conozco. }\end{array}$ & 275 & 1 & 5 & 1.09 & 1.86 & \\
\hline $\begin{array}{l}\text { Me envían solicitudes de amistad por redes sociales personas que } \\
\text { no conozco. }\end{array}$ & 275 & 1 & 5 & 1.18 & 1.89 & \\
\hline $\begin{array}{l}\text { Acepto solicitudes de amistad en redes socialdes de personas que } \\
\text { no conozco. }\end{array}$ & 275 & 1 & 5 & 1.14 & 1.92 & \\
\hline Me gusta platicar con personas desconocidas. & 275 & 1 & 5 & 2.08 & 1.67 & \\
\hline $\begin{array}{l}\text { Me gusta platicar con personas mayores porque me enseñan } \\
\text { cosas. }\end{array}$ & 275 & 1 & 5 & 1.12 & 1.79 & \\
\hline Se me hace interesante hablar con personas mayores que yo. & 275 & 1 & 5 & 1.16 & 1.74 & \\
\hline Me parece aburrido hablar con personas mayores que yo. & 275 & 1 & 5 & 1.14 & 1.92 & \\
\hline Evito hablar con personas mayores que yo. & 275 & 1 & 5 & 1.67 & 1.49 & \\
\hline Evito hablar con desconocidos. & 275 & 1 & 5 & 1.98 & 1.06 & \\
\hline $\begin{array}{l}\text { Comento en redes sociales estados de otras personas para que } \\
\text { me hablen. }\end{array}$ & 275 & 1 & 5 & 1.78 & 1.14 & \\
\hline $\begin{array}{l}\text { Me divierte leer comentarios de estados o publicaciones de otras } \\
\text { personas por redes sociales. }\end{array}$ & 275 & 1 & 5 & 2.02 & 1.86 & \\
\hline Envío a mis amigos publicaciones o estados que me dan risa. & 275 & 1 & 5 & 1.19 & 1.24 & \\
\hline Hago memes graciosos de amigos para reírnos. & 275 & 1 & 5 & 1.08 & 1.10 & \\
\hline Envío memes de amigos por redes sociales para divertirnos. & 275 & 1 & 5 & 1.68 & 1.06 & \\
\hline $\begin{array}{l}\text { Mis amigos me envían por redes sociales memes, publicaciones y } \\
\text { estados de otros compañeros de clase. }\end{array}$ & 275 & 1 & 5 & 1.14 & 1.23 & \\
\hline $\begin{array}{l}\text { Me envían contenido para adultos por redes sociales de } \\
\text { compañeros de clase o amigos. }\end{array}$ & 275 & 1 & 5 & 1.08 & 1.76 & \\
\hline $\begin{array}{l}\text { Me envían contenido para adultos por redes sociales de personas } \\
\text { que no conozco. }\end{array}$ & 275 & 1 & 5 & 1.04 & 1.69 & \\
\hline $\begin{array}{l}\text { Me envían contenidos para adultos por redes sociales de algún } \\
\text { familiar. }\end{array}$ & 275 & 1 & 5 & 1.09 & 1.78 & \\
\hline Dependencia de Internet y dispositivos móviles. & & & & & & .72 \\
\hline Uso Internet desde el hogar & 275 & 1 & 5 & 3.69 & 1.88 & \\
\hline
\end{tabular}




\begin{tabular}{|c|c|c|c|c|c|c|}
\hline Escala/ítem & $N$ & Min. & Max. & Media & D.E. & Alfa \\
\hline Uso Internet desde una computadora, laptop o tableta & 275 & 1 & 5 & 4.04 & 1.96 & \\
\hline Uso Internet con mi teléfono celular (smarthphone). & 275 & 1 & 5 & 4.16 & 1.85 & \\
\hline $\begin{array}{l}\text { Uso Internet desde cualquier lugar (escuela, cibercafé, casa de un } \\
\text { amigo, casa de un familiar, en lugares con conexión gratis). }\end{array}$ & 275 & & 5 & 4.08 & 1.89 & \\
\hline Uso Internet todos los días. & 275 & 1 & 5 & 4.12 & 1.68 & \\
\hline Duermo con el celular a un lado de la cama. & 275 & 1 & 5 & 4.04 & 1.12 & \\
\hline Cuando voy al baño, me llevo el celular. & 275 & 1 & 5 & 3.89 & 1.72 & \\
\hline Me desvelo usando el celular & 275 & 1 & 5 & 3.68 & 1.08 & \\
\hline No me doy cuenta del tiempo mientras estoy con el celular. & 275 & 1 & 5 & 4.10 & 1.98 & \\
\hline Le quito horas al sueño por usar el celular. & 275 & 1 & 5 & 3.45 & 1.12 & \\
\hline Me siento inquieto cuando no tengo el celular conmigo. & 275 & 1 & 5 & 3.22 & 1.04 & \\
\hline Me siento incomodo si no traigo conmigo el celular. & 275 & 1 & 5 & 3.14 & 1.88 & \\
\hline Traigo el celular todo el día conmigo. & 275 & 1 & 5 & 3.12 & 1.09 & \\
\hline El celular es parte de mi. & 275 & 1 & 5 & 2.86 & 1.79 & \\
\hline Sin el celular me siento solo(a). & 275 & 1 & 5 & 2.67 & 1.09 & \\
\hline Mis papás me regañan por estar todo el día con el celular. & 275 & 1 & 5 & 2.89 & 1.10 & \\
\hline Derechos fundamentales & & & & & & .67 \\
\hline $\begin{array}{l}\text { Cuando uso aplicaciones y redes sociales me piden mis datos } \\
\text { personales. }\end{array}$ & 275 & 1 & 5 & 1.67 & 1.92 & \\
\hline Tengo perfil o cuenta en redes sociales. & 275 & 1 & 5 & 3.21 & 1.09 & \\
\hline Cuando estoy en Internet digo lo que pienso. & 275 & 1 & 5 & 3.76 & 1.19 & \\
\hline Me expreso libremente en Internet. & 275 & 1 & 5 & 3.24 & 1.22 & \\
\hline Cuando uso Intenet veo lo que quiero. & 275 & 1 & 5 & 3.12 & 1.08 & \\
\hline Puedo usar el Internet cuando quiero. & 275 & 1 & 5 & 2.28 & 1.16 & \\
\hline
\end{tabular}




\begin{tabular}{|c|c|c|c|c|c|c|}
\hline Escala/Ítem & $N$ & Min. & Max. & Media & D.E. & Alfa \\
\hline Cuando estoy en Internet mis papás ven lo que veo. & 275 & 1 & 5 & 2.34 & 1.67 & \\
\hline Cuando uso Internet mis papás no ven lo que yo veo. & 275 & 1 & 5 & 2.98 & 1.06 & \\
\hline Mis papás me prohiben usar el Internet cuando no están en casa. & 275 & 1 & 5 & 2.76 & 1.24 & \\
\hline Mis papás me enseñan a usar el Internet. & 275 & 1 & 5 & 1.92 & 1.21 & \\
\hline Mis papás me preguntan cómo usar Internet. & 275 & 1 & 5 & 1.89 & 1.04 & \\
\hline Mis papás me dicen que el Internet es peligroso. & 275 & 1 & 5 & 1.26 & 1.14 & \\
\hline Conozco los riesgos y peligros de Internet. & 275 & 1 & 5 & 2.22 & 1.06 & \\
\hline Mis papás me dejan usar el Internet cuando estoy solo. & 275 & 1 & 5 & 2.67 & 1.19 & \\
\hline Mis papás me advierten de los riesgos y peligros de usar Internet & 275 & 1 & 5 & 2.45 & 1.11 & \\
\hline Mis papás me preguntan lo que hago en Internet & 275 & 1 & 5 & 2.24 & 1.04 & \\
\hline $\begin{array}{l}\text { Mis papás cambian claves y contraseñas para que no use el } \\
\text { Internet. }\end{array}$ & 275 & 1 & 5 & 1.72 & 1.09 & \\
\hline Mis papás quitaron el Internet en casa. & 275 & 1 & 5 & 1.84 & 1.12 & \\
\hline Mis papás me castigan con el uso de Internet. & 275 & 1 & 5 & 1.17 & 1.66 & \\
\hline Mis papás no están cuando uso el Internet. & 275 & 1 & 5 & 3.89 & 1.08 & \\
\hline $\begin{array}{l}\text { Mis papás me ayudan a buscar información en Internet para mis } \\
\text { tareas. }\end{array}$ & 275 & 1 & 5 & 3.24 & 1.12 & \\
\hline Tengo claves y contraseñas para usar el Internet & 275 & 1 & 5 & 3.04 & 1.68 & \\
\hline No tengo las claves y contraseñas para usar el Internet & 275 & 1 & 5 & 2.12 & 1.79 & \\
\hline
\end{tabular}

En la tabla 3. Las pruebas de Correlaciones de Pearson, permitió establecer la relación de los factores que intervienen en el modelo, en el que se obtuvo una relación significativa entre el factor de sociabilidad virtual y derechos fundamentales $\left(.467^{*}\right)$. Se encontró igualmente, que la variable de sociabilidad virtual se correlaciona altamente con la variable de exposición al riesgo (.608**); es decir, a mayor interacción en sus procesos de sociabilidad, mayor exposición al riesgo de los menores de edad. También se obtuvo una correlación entre la sociabilidad virtual y la dependencia de Internet $\left(.643^{*}\right)$, lo mismo que la variable de derechos fundamentales se correlaciona altamente con el factor dependencia de Internet (.604**). Por 
último, la variable de exposición al riesgo aparece fuertemente correlacionada con la dependencia de Internet $\left(.492^{* *}\right)$.

Tabla 3. Correlaciones entre las variables de estudio

\begin{tabular}{lcccc}
\hline & $\begin{array}{c}\text { Sociabilidad } \\
\text { virtual }\end{array}$ & $\begin{array}{c}\text { Derechos } \\
\text { fundamentales }\end{array}$ & $\begin{array}{c}\text { Exposición al } \\
\text { riesgo }\end{array}$ & $\begin{array}{c}\text { Dependencia de } \\
\text { Internet }\end{array}$ \\
\hline $\begin{array}{l}\text { Sociabilidad virtual } \\
\begin{array}{l}\text { Derechos } \\
\text { fundamentales }\end{array}\end{array}$ & 1 & - & - & - \\
$\begin{array}{l}\text { Exposición al riesgo } \\
\text { Dependencia de }\end{array}$ & $.608^{* *}$ & 1 & - & - \\
\begin{tabular}{l} 
Internet \\
\hline
\end{tabular} & $.643^{*}$ & $.625^{*}$ & 1 & - \\
\hline
\end{tabular}

** La correlación es significativa al nivel 0,01 (bilateral).

* La correlación es significante al nivel 0,05 (bilateral).

\subsection{Análisis estadístico del modelo estructural}

La Figura 1 muestra el modelo de ecuaciones estructurales. En él es posible apreciar que el factor de exposición al riesgo está formado por el acceso voluntario a contenidos inapropiados ( $P F=.22)$, acceso voluntario a conductas inadecuadas por terceras personas ( $P F=.26)$, acceso involuntario a contenidos inapropiados ( $\mathrm{PF}=.44)$, acceso involuntario a conductas inadecuadas por terceras personas $(P F=.27)$. El factor de derechos fundamentales se formó con sus respectivos indicadores: involucramiento de los padres en la digitalización ( $\mathrm{PF}=.46)$, protección a la intimidad del menor ( $\mathrm{PF}=.22)$, libre expresión y asociación $(P F=.44)$, acceso a la información $(P F=.68)$, y finalmente, con el indicador protección de los menores contra el abuso ( $P F=.29)$. Por su parte, el factor de dependencia de Internet se formó congruentemente con sus indicadores preocupación constante con un puntaje factorial ( $\mathrm{PF}=.29)$, apego a dispositivos móviles ( $\mathrm{PF}=.72$ ), y tiempo de uso (PF=.62). Por último, el factor de sociabilidad virtual se formó con los indicadores interacción virtual con los pares con un puntaje factorial (PF=.76), interacción virtual con los padres ( $\mathrm{PF}=.64)$, interacción virtual con personas desconocidas $(P F=.68)$, e interacción virtual con personas conocidas ( $P F=.59)$.

El factor de exposición al riesgo está impactando de forma directa y positiva con el factor de dependencia de Internet con un Coeficiente Estructural $(C E=.49)$ y con el factor de Derechos fundamentales $(C E=.42)$. A su vez, el factor exposición al riesgo está siendo fuertemente afectado por el factor de sociabilidad virtual ( $C E=.60)$; al mismo tiempo, el factor de sociabilidad virtual predice directa y positivamente al factor de dependencia de Internet (CE=.64). Como se puede apreciar cada uno de los puntajes factoriales, así como los coeficientes estructurales de las correlaciones fueron altamente significativos. Las unidades estadísticas adoptadas fueron el Índice de Ajuste Normado de Bentler-Bonet (BBNFI=.90), Índice de Ajuste No Normado (NNFI=.92), y el Índice de Ajuste Comparativo (CFI= .95), las cuales permiten determinar la pertinencia y la interrelaciones entre variables propuestas para el modelo (Bentler, 2006). Los 
indicadores en cada una de las pruebas debía ser superior a .90; por su parte la Raíz Cuadrada del Cuadrado Medio del Error de Aproximación (RMSEA) fue de .05, resultando dentro de los parámetros aceptables, mientras que la Chi cuadrada fue de 16.24 y 18 grados de libertad (gI), con .12 de probabilidad asociada y finalmente, una $R^{2} .48$, lo cual significa que el modelo de ecuaciones estructurales explica en su conjunto el $48 \%$ de la varianza de los factores que intervienen en la exposición al riesgo asociados a los derechos fundamentales de los menores.

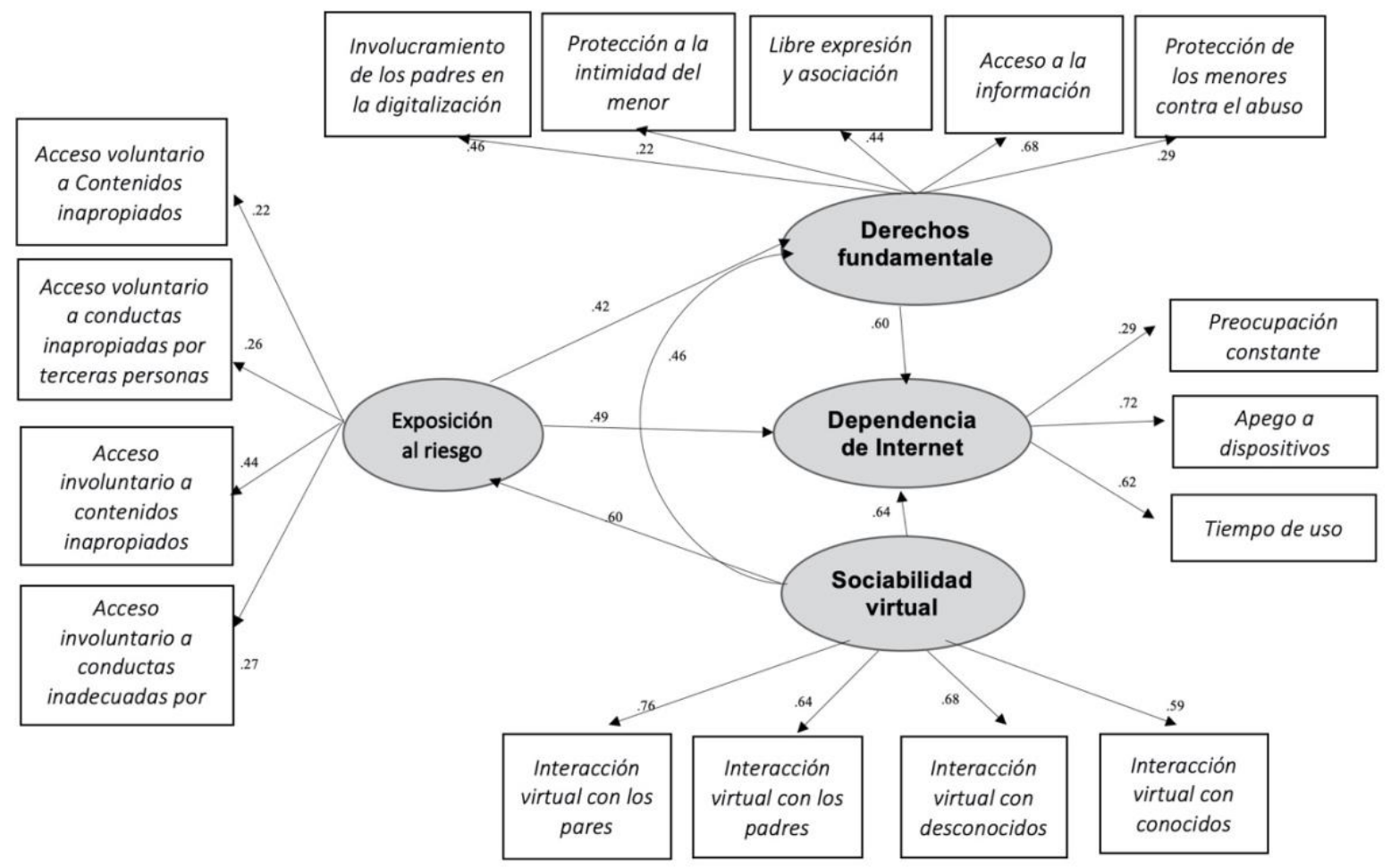

Figura 1. Modelo estructural. $\mathrm{N}=275, \mathrm{X} 2=16.24 ; \mathrm{GL}=18 ; \mathrm{P}=.12 ; \mathrm{BBNFI}:=.90 ; \mathrm{BBNNFI}:=.92 \mathrm{CFI}:=.95 ; \mathrm{RMSEA}=$ $.05 ; \mathrm{R} 2.48$.

\section{Discusión y conclusiones}

A la luz de los resultados y evidencias recogidas, se puede determinar que se dispuso de un instrumento fiable con datos consistentes en la validez del constructo. No solo fue posible constatar que el cuestionario aplicado de acuerdo con la teoría estadística cumple con una consistencia interna, sino que los 80 ítems derivados de las cuatro escalas analizadas para las diversas pruebas permitieron establecer un modelo de ecuaciones estructurales que comunicara el riesgo y la dependencia de Internet frente a los derechos fundamentales en la era digital de la infancia intermedia, particularmente en dos contextos: México y España. En este sentido, la batería compuesta por sus cuatro escalas que conforma al instrumento de exposición al riesgo y dependencia de Internet frente a los derechos fundamentales de los 
menores es útil para estudios diagnósticos de sujetos en un rango de edad aproximada de entre 10 y 13 años.

En estricto sentido, el cuestionario permite establecer relaciones empíricas explicativas a partir de su fundamento teórico y el análisis estadístico factorial. En su conjunto, el estudio descriptivo y correlacional, permitió, adicionalmente, exponer un modelo estructural que diera cuenta de la exposición al riesgo frente a los derechos fundamentales y la dependencia de Internet por parte de los menores en etapa de infancia intermedia. De los resultados esperados del modelo estructural se pudo demostrar que el factor de exposición al riesgo tiene una influencia directa y positiva con el factor de dependencia de Internet y con el factor de derechos fundamentales. A partir de estas correlaciones, es posible determinar que los menores cuando acceden de manera voluntaria e involuntaria a conductas inadecuadas por terceras personas y contenidos inapropiados existen una mayor predisposición directa y positiva a afectar algunos de sus derechos fundamentales, tales como la protección a la intimidad y en un sentido estricto, la protección de los menores contra el acoso y el abuso. Asimismo, se advierte que existe una relación significativa entre la exposición al riesgo con el uso excesivo y/o dependencia de Internet, incrementando el tiempo de uso y el apego a los dispositivos en los infantes.

Como aspecto positivo, habría que resaltar que el modelo exhibe un mayor involucramiento por parte de los padres en la digitalización del menor, en clara sintonía con uno de los derechos universales del infante. Sin duda se habla de una generación de padres y madres más responsables de lo que hacen sus hijos frente al uso de las tecnologías y dispositivos móviles. Sin embargo, debe tenerse en cuenta que los menores siguen mostrando un mayor liderazgo y dominio de las tecnologías digitales que sus padres. Si bien el modelo comunica y advierte de una exposición al riesgo frente a los derechos fundamentales y la dependencia de Internet, la misma exposición deviene por una afectación directa del factor de sociabilidad virtual.

En estudios previos se ha evidenciado esta correlación, y en consecuencia se ha discutido seriamente que las generaciones interactivas de hoy en día se caracterizan primordialmente por los procesos de sociabilidad en línea (Contreras y Campa, 2017; Contreras, 2019), Según la evidencia recogida del modelo estructural, los menores en infancia intermedia gustan preferentemente de relacionarse con sus iguales como parte de los procesos de sociabilidad por redes sociales y múltiples plataformas que les permiten interactuar entre ellos y al mismo tiempo con personas conocidas y desconocidas.

Como toda investigación de esta índole, el estudio presenta algunas limitaciones. La muestra que se seleccionó fue a partir de criterios de inclusión y exclusión específicos. Se aplicó solo a menores en un rango de edad. En lo referente al modelo estructural, se debe señalar que no se consideraron los 10 derechos de la infancia en la era digital que propone UNICEF, sino que la selección de los 5 derechos fundamentales que se correlacionan en el presente modelo se hizo con fundamentos de estudios diagnósticos previos con enfoques mixtos, en la que evidenciaba ya, una línea muy específica a indagar en relación con la propuesta del modelo establecido. Por lo tanto, se sugiere que en próximas investigaciones además de considerar una mayor representatividad de la muestra en ambos contextos, se incluyan otros derechos fundamentales como los que señala UNICEF. 


\section{AGRADECIMIENTOS:}

La investigación está financiada con una beca del Programa para el Desarrollo Profesional Docente (Prodep - tipo superior). Secretaría de Educación Pública (SEP). Referencia del proyecto 0704256. Unison-PTC 275.

\section{REFERENCIAS}

Bergmark, K., Bergmark, A., \& Findahl, O. (2011). Extensive internet involvement-addiction or emerging lifestyle? International Journal of Environmental Research and Public Health, 8(12), 4488-4501. https://doi:10.3390/ijerph8124488

Bentler, P. M. (2006). EQS 6, Structural Equations Program Manual. Encino, CA: Multivariate Software Inc. http://www.econ.upf.edu/ satorra/CourseSEMVienna2010/EQSManual.pdf

Byrne, J. D. Kardefelt-Winther, S. Livingstone and M Stoilova (2016) Global Kids online research synthesis, 2015-2016. UNICEF office of Research Innocenti and London School Economics and Political Sciences. https://www.unicefirc.org/publications/pdf/IRR_2016_01.pdf

Chapman Quevedo, W., (2015) El Concepto de Sociabilidad como referente del Análisis hiistórico. Investigación y Desarrollo, 23, no. 1. http://dx.doi.org/10.14482/indes.23.1.6040

Contreras Cázarez, C.R. (2019a) Validación de la escala de derechos fundamentales y uso de redes sociales en menores de escuelas primarias públicas en México, en Aída María de Vicente Domínguez y Javier Sierra Sánchez (Coords.). Aproximación periodística y educomunicativa al fenómeno de las redes sociales. Editorial McGraw Hill. Madrid, España.

Contreras Cázarez, C. R. (2019b) Proceso de socialización en jóvenes de secundarias en el acceso y uso de Internet a partir de un modelo estructural. Educación, Lenguaje y Sociedad EISSN 2545-7667 Vol. XVI № 16 (Abril 2019) pp. 1-28 DOI:http://dx.doi.org/10.19137/els-2019-161604. Concultado el 16 de junio de 2019 en: https://cerac.unlpam.edu.ar/index.php/els/article/view/3864/3921.

Contreras Cázarez, C.R. y León Duarte, GA. (2019) Análisis factorial de un modelo de socialización y confianza en la dependencia de Internet. Revista Electrónica de Investigación Educativa. 21, e25, 1-13. https://doi.org/10.24320/redie.2019.21.e25.2112

Contreras Cázarez, C. R., Campa Álvarez, R. (2017). Caracterización del perfil de los estudiantes de secundarias en el acceso y uso de internet a partir de las TIC. EDUTEC, Revista $\begin{array}{llll}\text { Electrónica de } & \text { Tecnología }\end{array}$ 
http://www.edutec.es/revista/index.php/edutec-e/article/view/941. http://dx.doi.org/10.21556/edutec.2018.61.

Craig, R. T. (2013). Constructing theories in communication research. Theories and models of communication, 1, 39-57.

Cronbach, L. J. (1984). Essentials of psychological testing (4th ed.). New York, NY: Harper \& Row.

Del Moral, M.E. y Villalustre, L. (2012). Presencia de los futuros maestros en las redes sociales y perspectivas de uso educativo. Revista Latinoamericana de Tecnología Educativa (RELATEC). 11(1), 41-51. Recuperado de http://relatec.unex.es/article/viewFile/843/633

Davis, R. A. (2001). A cognitive-behavioral model of pathological Internet use. Computers in human behavior, 17(2), 187-195. https://doi.org/10.1016/S0747-5632(00)00041-8

Echeburúa E, \& Corral P. (2009) Adicciones psicológicas: más allá de la metáfora. Clínica y Salud. 1994; $5(251-258)$. https://journals.copmadrid.org/clysa/art/07e1cd7dca89a1678042477183b7ac3f

Echeburúa, E. \& de Corral, P. (2010). Adicción a las nuevas tecnologías ya las redes sociales en jóvenes: un nuevo reto. Adicciones: Revista De Socidrogalcohol, 22 (2), pp. 91-96. http://www.adicciones.es/index.php/adicciones/article/view/196/186

Fondo de las Naciones Unidas para la Infancia (UNICEF)(2018). Annual results report 2018. Office of Research - Innocenti.

Fondo de las Naciones Unidas para la Infancia (UNICEF)(2017). The State of the World's Children 2017: Children in a Digital World. https://www.unicef.org/publications/index_101992.html

Fondo de las Naciones Unidas para la Infancia (UNICEF)(2014). Convención sobre los derechos del niño. https://www.unicef.es/educa/biblioteca/convencion-sobre-derechos-nino

Fondo de las Naciones Unidas para la Infancia (UNICEF) (2005). Vigía de los derechos de la niñez mexicana. Número 2, Año $1 . \quad$ La edad escolar. https://www.unicef.org/mexico/spanish/mx_resources_vigia_II.pdf

Fernández Pérez, A., (2016). La protección de los derechos fundamentales de los menores en Internet desde la perspectiva europea. Ius et Praxis [en linea] 2016, 22. https://scielo.conicyt.cl/pdf/iusetp/v22n1/art11.pdf

Fortson, B. L., Scotti, J. R., Chen, Y. C., Malone, J., \& Del Ben, K. S. (2017). Internet use, abuse, and dependence among students at a southeastern regional university. Journal of American College Health, 56(2), 137-144. https://doi.org/10.3200/JACH.56.2.137-146

Gómez, J.A. y Redondo, C. (2011). Las redes sociales como fuente de conocimiento en la enseñanza primaria. XII Congreso Internacional de Teoría de la Educación (CITE) 2011, 
Barcelona. Texto completo recuperado de http://www.cite2011.com/Comunicaciones/TIC/150.pdf

Hernández, R., Fernández, C., \& Baptista, P. (2010). Metodología de la investigación. México: Mc Graw Hill.

Ibáñez Zapata, P., \& Sánchez Vera, M. (2019). Percepción de los docentes sobre el uso de las redes sociales en el aula de Primaria. Una experiencia en un centro educativo de la Región de Murcia. Edutec. Revista Electrónica De Tecnología Educativa, (66). https://doi.org/10.21556/edutec.2018.66.1219

Instituto Nacional de Estadística y Geografía (INEGI) (2018). Estadísticas a propósito del día mundial del Internet. https://www.inegi.org.mx/contenidos/saladeprensa/aproposito/2018/internet2018_N al.pdf

Kaiser, H. F. (1958). The varimax criterion for analytic rotation in factor analysis. Psychometrika, 23(3), 187-201.

Kuss, D. J., \& Lopez-Fernandez, O. (2016). Internet addiction and problematic Internet use: A systematic review of clinical research. World journal of psychiatry, 6(1), 143. http://doi:10.5498/wjp.v6.i1.143

León-Duarte, G; Contreras Cázarez, CR; Moreno Carrillo, D. (2016): Probando modelos interdisciplinares inclusivos en la dependencia de Internet en Jóvenes. Nuevas variables asociadas||. Revista Latina de Comunicación Social, 71, pp. 616 a 631 . Recuperado de http://www.revistalatinacs.org/071/paper/1112/RLCS-paper1112.pdf.

León-Duarte, G; Caudillo R. D.; Contreras-Cázarez, C., Moreno C. D. (2015) Jóvenes y medios digitales móviles en México. Un estudio de variables asociadas en perspectiva Interdisciplinar. Primera edición. PEARSON EDUCACIÓN. México.

León-Duarte, G; Caudillo, D.Y., Contreras-Cazarez, C.R. y Moreno, E. (2014). Internet seguro y jóvenes de secundaria en México. Hermosillo: Universidad de Sonora.

Lin, S. S., \& Tsai, C. C. (2002). Sensation seeking and internet dependence of Taiwanese high school adolescents. Computers inhuman behavior, 18(4), 411-426. https://doi.org/10.1016/S0747-5632(01)00056-5

Livingstone, S., Mascheroni, G., \& Staksrud, E. (2018). European research on children's internet use: Assessing the past and anticipating the future. New Media \& Society, 20(3), 11031122. https://doi.org/10.1177/1461444816685930

Livingstone, S., Haddon, L., Görzig, A., \& Ólafsson, K. (2017). with members of the EU Kids Online Network.(2011). Risks and safety on the internet, 9-16. http://eprints.Ise.ac.uk/33731/ 
Lozano-Vicente, A. (2016). Los derechos del niño: cuestiones sobre su fundamentación. Revista Latinoamericana de Ciencias Sociales, Niñez y Juventud, 14 (1), pp. 67-79. https://doi:10.11600/1692715x.1413290515

Montag, C., \& Reuter, M., (2017) Internet Addiction. Neuroscientific Approaches and Therapeutical Implications Including Smartphone Addiction. Ed. Springer. ISBN: 9783319462752.

Rojas Jara, C., Ramos Vera, J., Pardo-González, E., y Henríquez-Caroca, F. (julio-diciembre, 2018). Adicción a internet en adolescentes: una breve revisión. Drugs and Addictive Behavior, 3(2), 267-281. http://dx.doi.org/10.21501/24631779.2876

Simkin, H., y Becerra, G., (2013) El proceso de socialización. Apuntes para su exploración en el campo psicosocial. Ciencia, Docencia y Tecnología. Vol. XXIV. No. 47. pp.119-142. https://dialnet.unirioja.es/servlet/articulo?codigo $=4696738$

Vondrácková, P., \& Gabrhelík, R. (2016). Prevention of internet addiction: A systematic review. Journal of Behavioral Addictions, 5 (4), 568-579. Doi: https://doi.org/10.1556/2006.5.2016.085.

Young, KS (1998). Adicción a Internet: La aparición de un nuevo trastorno clínico. CyberPsychology \& Behavior, 1, 237-244. http://dx.doi.org/10.1089/cpb.1998.1.237

Young K. S. y Rodger, R. C. (1998). "The Relationship Between Depression and Internet Addiction". http://www.netaddiction.com/articles/cyberpsychology.pdf

\section{Para citar este artículo:}

Contreras Cázarez, C. R., León Duarte, G. A., y Zozaya Durazo, L. D. (2020). Variables predictoras de riesgo frente a los derechos del infante en la era digital. Un estudio de México y España. Edutec. Revista Electrónica De Tecnología Educativa, (73), 122-139. https://doi.org/10.21556/edutec.2020.73.1549 